Ophthalmologe 2012 · 109:217-218

DOI 10.1007/s00347-012-2579-y

๑) Springer-Verlag 2012
F.G. Holz ${ }^{1}$ B. Knapp ${ }^{2}$

${ }^{1}$ Universitäts-Augenklinik Bonn

${ }^{2}$ Springer-Verlag, Heidelberg

\title{
Der Ophthalmologe - digitale Optionen ergänzen die Zeitschrift sinnvoll
}

Liebe Leserinnen, liebe Leser,

die Zeitschrift Der Ophthalmologe als Organ der Deutschen Ophthalmologischen Gesellschaft (DOG) blickt auf eine lange Tradition zurück. Im Jahr 2012 erscheint bereits der 109. Band der Zeitschrift, und es sind weit mehr als 5000 DOG-Mitglieder, die Der Ophthalmologe Monat für Monat über alle wichtigen Entwicklungen in der Augenheilkunde auf dem Laufenden hält.

In den letzten Jahren veränderte sich das wissenschaftliche Publikationswesen grundlegend. Die Möglichkeit der digitalen Verarbeitung und Verbreitung wissenschaftlicher Erkenntnisse über das Internet hat dazu geführt, dass auch die Zeitschrift Der Ophthalmologe heute weit mehr ausmacht als allein die Veröffentlichung in Druckform.

Bereits für die Einreichung und Begutachtung von Manuskripten für Der Ophthalmologe steht den Autoren und Gutachtern das onlinebasierte Manuskripteinreichungssystem Editorial Manager zu Verfügung. Dieses hat sich in nunmehr 5 Jahren fest etabliert und hat entscheidend dazu beigetragen die Dauer des Peer-Reviews zu verkürzen und den $\mathrm{Pu}$ blikationsprozess zu beschleunigen. Das Feedback von Autoren und Gutachtern ist dabei durchweg positiv.

Die für die Zeitschrift angenommenen Manuskripte werden nicht nur in der Printversion von Der Ophthalmologe publiziert, sondern auch in elektronischer Form in der Datenbank SpringerLink (www.springerlink.com) veröffentlicht und archiviert. Das elektronische Archiv der Zeitschrift umfasst mittlerwei- le sämtliche Ausgaben seit 1997, d. h. insgesamt 15 Bände, 180 Ausgaben und mehr als 2500 einzelne Beiträge. Die elektronische Publikation auf SpringerLink erlaubt jedem Autor auch Zusatzmaterialien, z. B. Videosequenzen, zu seinem Beitrag zur Verfügung zu stellen.

\section{\) Das elektronische Volltextarchiv der Zeitschrift umfasst derzeit mehr als 2500 einzelne Beiträge}

Neben der Archivierung der laufenden Ausgaben werden auf SpringerLink ebenfalls Beiträge nach dem „Online-FirstPrinzip“ veröffentlicht. Das heißt, der Leser findet hier Beiträge, die zur Publikation angenommen wurden, aber noch nicht im gedruckten Heft erschienen sind. Ab dem Zeitpunkt der Online-first-Veröffentlichung gilt der Beitrag als publiziert und ist über den angegebenen DOI zitierfähig.

Der Zugriff auf sämtliche elektronische Volltexte ist im Abonnement der Zeitschrift Der Ophthalmologe inbegriffen. Darüber hinaus ist der Zugang weltweit in vielen Instituten und Bibliotheken möglich, in Deutschland u. a. in allen Universitätsklinken und Max-Planck-Instituten. Für DOG-Mitglieder ist der Zugriff ebenfalls von der Homepage der DOG (www. dog.org) aus dem geschlossenen Mitgliederbereich ohne erneute Registrierung möglich. Im Jahr 2012 ist darüber hinaus auch das englischsprachige Publikationsorgan der DOG, Graefe's Archive for Clinical and Experimental Ophthalmology, für alle DOG-Mitglieder frei über die DOGHomepage verfügbar.

Die Zugriffszahlen auf die elektronischen Inhalte von Der Ophthalmologe zeigen, dass von Jahr zu Jahr mehr Leser diese Form der Informationsbeschaffung nutzen. Derzeit werden jeden Monat durchschnittlich 6000 Artikel der Zeitschrift im Volltext aufgerufen, fast doppelt so viele wie noch vor 3 Jahren. Auf der Zeitschriften-Homepage www.DerOphthalmologe.de kann jeder mit verfolgen, wie viele und welche Beiträge aus Der Ophthalmologe in den letzten 7, 30 und 90 Tagen von SpringerLink heruntergeladen wurden. Die 5 am häufigsten heruntergeladenen Beiträge sind dabei grundsätzlich auch für Nichtabonnenten der Zeitschrift frei verfügbar.

\section{》) Die Online-CME-Fort- \\ bildung wird insbesondere von niedergelassenen Augenärzten gerne genutzt}

Auch die Teilnahme an den CME-Fortbildungen von Der Ophthalmologe erfolgt bereits seit mehreren Jahren ausschließlich online über das CME-Portal CME. springer.de. Die Anzahl der Teilnahmen an den Weiterbildungseinheiten aus Der Ophthalmologe steigt jährlich. Über die Auswertung der CME-Evaluationsbögen wissen wir, dass diese Möglichkeit der Online-Fortbildung insbesondere von niedergelassenen Augenärzten sehr gerne genutzt wird. Zudem hat sich gezeigt, dass fast $90 \%$ der Teilnehmer nach der Lektü- 


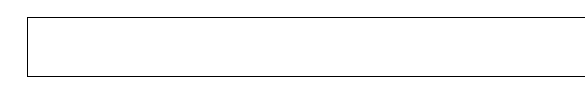

re des CME-Beitrags die gewonnenen Erkenntnisse in der Praxis umsetzten, wobei sogar ein Drittel der Teilnehmer seine bisherige Strategie verändert. Dies ist für uns Bestätigung und Ansporn zugleich. Wir fühlen uns hier in der Verantwortung, auch in Zukunft unser besonderes Augenmerk auf die Qualität der CMEBeiträge zu legen und unseren Lesern in dieser Rubrik auch weiterhin praxisnahe Beiträge auf fachlich hohem Niveau zu bieten. In den kommenden Monaten wird sich das Gesicht der CME-Fortbildung bei Springer noch einmal grundlegend wandeln. Jeder Beitrag wird dann in einer neuen, speziellen Onlinefassung zur Verfügung stehen, was auch die Einbindung von interaktiven Elementen sowie zusätzlichem Bild- und Videomaterial erlaubt.

Wir freuen uns sehr, wenn unsere $\mathrm{Au}$ toren, unsere Gutachter und vor allem unsere Leser von den digitalen Optionen der Zeitschrift profitieren und diese nutzen. Wir wünschen Ihnen auch in Zukunft eine anregende Lektüre mit Der Ophthalmologe!

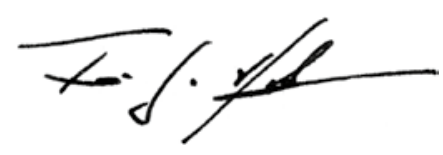

F.G. Holz

Herausgeber Der Ophthalmologe

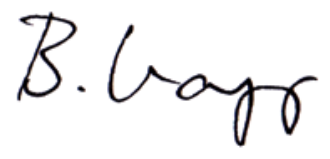

B. Knapp

Redaktionsleitung Der Ophthalmologe

\section{Korrespondenzadressen}

\section{Prof. Dr. F.G. Holz}

Universitäts-Augenklinik Bonn

Ernst-Abbe-Str. 2, 53127 Bonn

frank.holz@ukb.uni-bonn.de

\section{B. Knapp}

Springer-Verlag

Tiergartenstraße 17, 69121 Heidelberg

bettina.knapp@springer.com

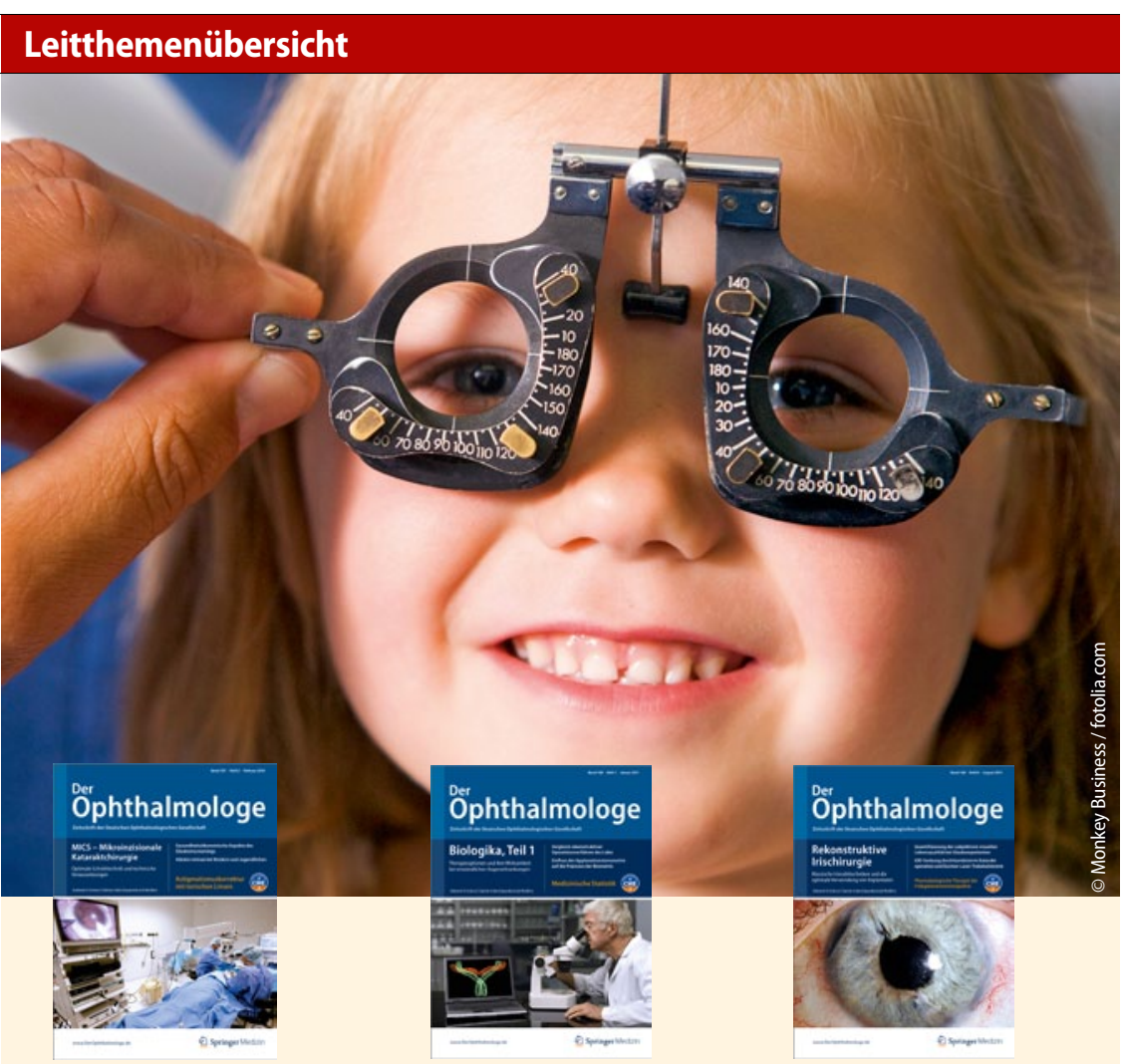

\section{Der Ophthalmologe bietet Ihnen jeden Monat umfassende und aktuelle Beiträge zu interessanten Themenschwerpunkten aus allen Bereichen der Ophthalmologie.}

Möchten Sie ein bereits erschienenes Heft nachbestellen? Einzelne Ausgaben können Sie direkt bei unserem Kundenservice zum Preis von je EUR 35,- zzgl. Versandkosten beziehen:

\section{ÜBERBLICK}

2011

01/11 Biologika, Teil 1

02/11 Venöse retinale Gefäßverschlüsse

03/11 Biologika, Teil 2

04/11 Fallstricke der Brillenbestimmung

05/11 Orbitaerkrankungen, Teil 1

06/11 Orbitaerkrankungen, Teil 2

07/11 Glaukom im Kindesalter

08/11 Rekonstruktive Irischirurgie

09/11 Perspektiven der Hornhauttransplantation

10/11 Verätzungen und Verbrennungen des Auges

11/11 Okuläre Hypertension

12/11 Augenschmerzen aus interdisziplinärer Sicht

So erreichen Sie unseren Kundenservice:

Springer Customer Service Center GmbH Kundenservice Zeitschriften

Haberstr. 7

69126 Heidelberg

Tel.:+49 $6221345-4303$

Fax:+49 $6221345-4229$

E-Mail:leserservice@springer.com

\section{www.DerOphthalmologe.de}

\section{VORSCHAU}

2012

01/12 Smartphones in der Augenheilkunde

02/12 Therapieansätze bei erblichen Netzhautdystrophien

03/12 Ophthalmologische Therapie in der Schwangerschaft

04/12 Funktionelle Glaukomdiagnostik

05/12 Lidchirurgie

06/12 Morbus Behcet 